\title{
Measurement of Bepicolombo Mission Medium Gain Antenna Parameters
}

\author{
Victor Sánchez, Aingeru Barrio, Fernando Martín, Iñaki Pinto, Rafael García , Leandro de Haro , José Luis \\ Besada, Belén Galocha, Manuel Sierra Castañer
}

\begin{abstract}
This paper is aimed to explain the radiation test in temperature performed on the radiofrequency assembly (RFA) of the Medium Gain Antenna (MGA) of ESA BepiColombo mission. The goal of this mission is to observe and study Mercury and its surroundings in a very demanding and stringent environment in terms of temperature and radiation. The paper presents the measurement set-up for the qualification campaign of the antenna, where it was necessary to characterize the antenna in a representative thermal environment.
\end{abstract}

Index Terms-antenna measurement, temperature, space applications.

\section{INTRODUCTION}

BepiColombo ESA mission [1] has the goal of observing and studying Mercury and its surroundings. The spacecraft and the antennas will work in an extreme environment from temperature and radiation point of view. This paper describes the set-up and test procedure of the Medium Gain Antenna (MGA) Major Assembly radiation test in temperature. This antenna is an X-Band dual flare horn steerable in two degrees of freedom, which provides two-way communications in safe mode between the spacecraft and Earth. The Medium Gain Antenna Major Assembly (MGAMA), shown in Fig. 1, works as backup of the High Gain Antenna (HGA) and also at several conditions of the cruise to Mercury and the mission phase. SENER is responsible for the MGAMA assembly, integration and test, and also design of the Antenna Pointing Mechanism (APM), MGAMA boom, Hold Down Release Mechanism (HDRM), and APM Electronics (APME). The radiofrequency assembly (which includes boom waveguides and horn antenna) is designed and manufactured by RYMSA ESPACIO. The MGA Rotary Joint Assembly (RJA) is responsibility of COBHAM. In order to minimize overall insertion loss both RFA and RJA are manufactured in silver and gold plated titanium respectively.

The authors from UPM want to acknowledge the Madrid Region Government project for financing the project Space Debris Radar (S2013/ICE-3000 SPADERADAR-CM) and the Spanish Government, for the support of the project ENABLING5G "Enabling Innovative Radio Technologies for 5G networks" (TEC2014-55735-C3-1-R)

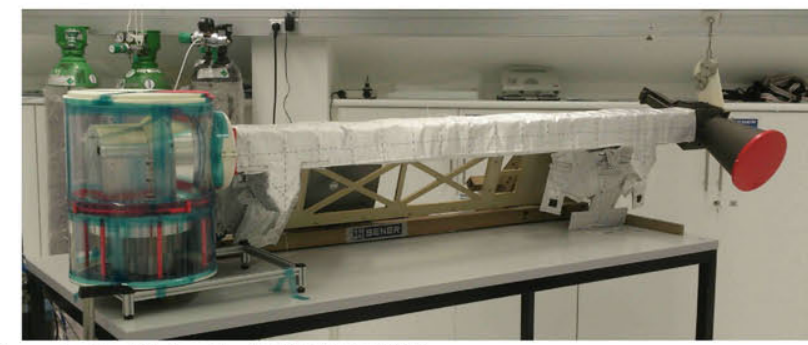

Figure 1. BepiColombo MGAMA EQM

The MGA-RFA was measured in the LEHA-UPM facilities (Antenna Measurement Laboratory of Technical University of Madrid) in Spain.

This paper is divided in the following sections: Section II explains the test plan, Section III the test set up and Section IV the test results. Finally, Section V includes the conclusions of the paper.

\section{MGA TEST PLAN}

From the engineering point of view, one of the most challenging points of BepiColombo mission is to withstand the extremely demanding thermal environment of Mercury. This is considered in the design of almost all the spacecraft subsystems, although it becomes especially critical in the case of those instruments and systems that are directly exposed to solar flux, like the antenna subsystem. Consequently, it was necessary to quantify and evaluate how the RF performances would evolve as function of conductivity variations and thermal distortions in a representative thermal environment. Thus, both the insertion loss and the radiation pattern had to be characterized in temperature.

In order to provide an accurate extrapolation of the thermal behavior of RFA, the measurements were performed at the six most significant frequencies: $7145 \mathrm{MHz}, 7165 \mathrm{MHz}$ and 7190 $\mathrm{MHz}$ at uplink band; and $8400 \mathrm{MHz}, 8425 \mathrm{MHz}$ and 8450 $\mathrm{MHz}$ at downlink band. The maximum test temperature was initially set to $200^{\circ} \mathrm{C}$. However, due to thermal limitations of the SMA cables used, the maximum temperature of the test was finally set to $150^{\circ} \mathrm{C}$. This temperature allows obtaining accurate extrapolations of the antenna behavior at the maximum foreseen operating temperature (over $530^{\circ} \mathrm{C}$, obtained by analysis). Measurements were performed at ambient room temperature $\left(23^{\circ} \mathrm{C}\right), 100^{\circ} \mathrm{C}, 120^{\circ} \mathrm{C}$ and $150^{\circ} \mathrm{C}$. 
Radiation patterns were acquired within a $\pm 60^{\circ}$ angular range. Axial ratio and VSWR were also monitored.

\section{MGA TEST SETUP}

As it was mentioned before, the MGA was measured in the antenna measurement facilities of the Technical University of Madrid [2]. Since heating up the entire anechoic chamber is not possible in order to reproduce the required thermal environment, an alternative solution was developed: design, manufacture and build a oven where the full RFA could be installed, isolated from the rest of the anechoic chamber. This allows heating up the antenna and boom waveguides without inducing distortions from the set up. The oven is depicted in Fig. 2.

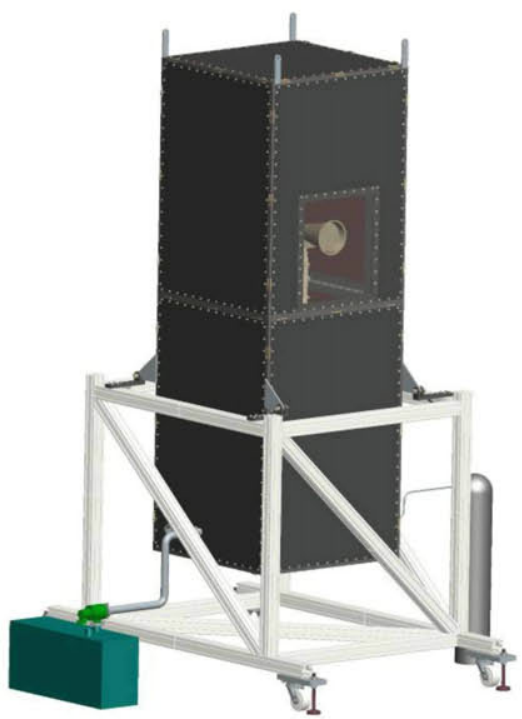

Figure 2. RF oven overview

The internal dimension of the oven were $3 \mathrm{~m} \times 1 \mathrm{~m} \times 1 \mathrm{~m}$. The structure is made of stainless steel and divided into two differenced areas. The upper one contains the dual flare horn and septum polarizer, which are made of bare titanium. The lower one contains boom waveguides -which are made of silver plated titanium-. In order to prevent oxidation of the silver coating at high temperatures, an inert atmosphere is requested in this area. Oxygen is removed with vacuum pump, and replaced by Argon. This area is sealed from outside and the upper part of the oven with Teflon gaskets, which allow RF propagation. The gasket is tightly fastened in both waveguide flanges, avoids the air entering into the silver plated titanium waveguide and also the Argon going out the oven. In one of the walls of the upper area, and close to the horn aperture it was placed a Kapton RF transparent window of $600 \mathrm{~mm} \times 500 \mathrm{~mm}$. This makes performing antenna measurements possible while keeping the required thermal environment inside. The rest of the oven internal walls are metallic. The outside was covered with insulating plates (except the RF window), which minimizes thermal losses and allows maintaining the oven at the commanded temperature.
The bottom of the RF oven has a stainless steel flexible circular plate in order to correctly attach the antenna under test to the external measurement RF waveguide, getting thermal isolation and absorbing boom dilations due to temperature increase. A short Titanium waveguide section is placed between the RF oven outer wall and a final stainless steel solid waveguide with UBR 84 flange, in order to avoid heat reaching the measurement RF test cables.

The tilting of the horn due temperature is controlled with two lasers that are attached to the outer titanium waveguide and pointed in two different directions. Their beams impinge to calibrated surfaces placed in supports that located at large distance. Measuring distances in the calibrated surfaces along the different temperatures, the tilting of the antenna assembly can be derived.

$\mathrm{RF}$ reflections due to the oven influence are minimized. This is achieved by coating with RF absorber the metallic face of the oven which is in front of the measurement probe. The reflectivity of this absorber must be at lower than $-20 \mathrm{~dB}$ to avoid reflecting signals reaching the probe and distorting the measurements. Fig. 3 shows the RF setup installed in the planar near field system of Technical University of Madrid without the Kapton RF window.

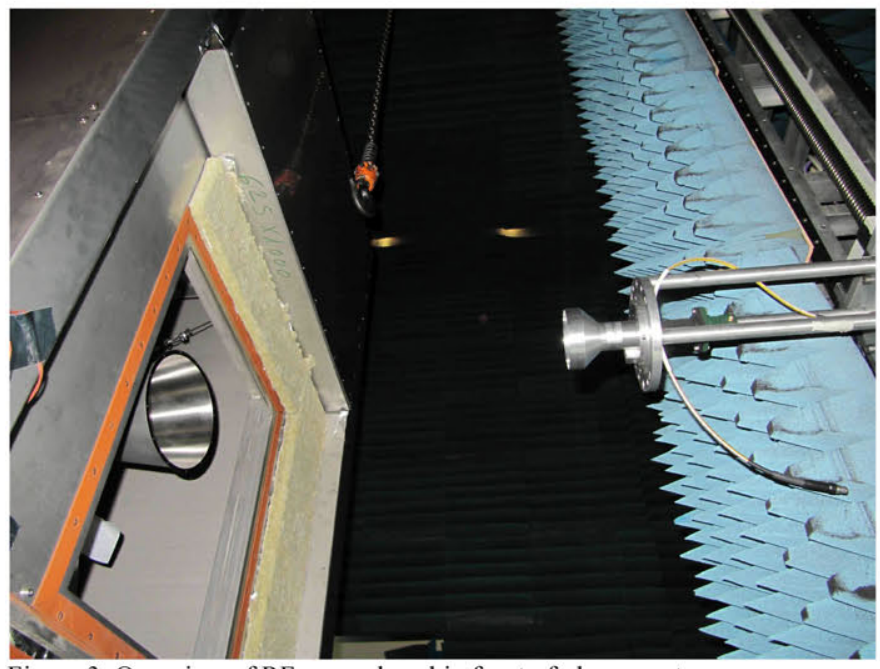

Figure 3. Overview of RF oven placed in front of planar system

\section{MGA TEST RESUlts}

Table I shows some of the gain results of the MGA antenna [3-4]. They are compared with the results obtained by the Antenna Manufacturer (RYMSA ESPACIO) once applied the extrapolation in temperature.

TABlE I. Antenna Gain MeAsurement Results $\left(100,120,150^{\circ} \mathrm{C}\right)$

\begin{tabular}{|l|c|c|}
\hline & $\begin{array}{c}\text { RYMSA } \\
\text { EXTRAPOLATED }\end{array}$ & $\begin{array}{c}\text { M4: } \\
100^{\circ} \mathrm{C}\end{array}$ \\
\hline F1: $7.145 \mathrm{GHz}$ & 20.99 & 21.04 \\
\hline F2: $7.165 \mathrm{GHz}$ & 21.04 & 21.14 \\
\hline F3: $7.190 \mathrm{GHz}$ & 21.08 & 21.07 \\
\hline F4: $8.400 \mathrm{GHz}$ & 22.47 & 22.52 \\
\hline F5: $8.425 \mathrm{GHz}$ & 22.54 & 22.39 \\
\hline F6: $8.450 \mathrm{GHz}$ & 22.62 & 22.49 \\
\hline
\end{tabular}




\begin{tabular}{|l|c|c|}
\hline & $\begin{array}{c}\text { RYMSA } \\
\text { EXTRAPOLATED }\end{array}$ & $\begin{array}{c}\text { MC: } \\
120^{\circ} \mathrm{C}\end{array}$ \\
\hline F1: $7.145 \mathrm{GHz}$ & 20.97 & 20.81 \\
\hline F2: $7.165 \mathrm{GHz}$ & 21.02 & 20.76 \\
\hline F3: $7.190 \mathrm{GHz}$ & 21.06 & 20.81 \\
\hline F4: $8.400 \mathrm{GHz}$ & 22.45 & 22.57 \\
\hline F5: $8.425 \mathrm{GHz}$ & 22.52 & 22.63 \\
\hline F6: $8.450 \mathrm{GHz}$ & 22.60 & 22.49 \\
\hline
\end{tabular}

\begin{tabular}{|l|c|c|}
\hline & $\begin{array}{c}\text { RYMSA } \\
\text { EXTRAPOLATED }\end{array}$ & $\begin{array}{c}\text { M5: } \\
150^{\circ} \mathrm{C}\end{array}$ \\
\hline F1: $7.145 \mathrm{GHz}$ & 20.95 & 20.98 \\
\hline F2: $7.165 \mathrm{GHz}$ & 21.00 & 21.07 \\
\hline F3: $7.190 \mathrm{GHz}$ & 21.04 & 21.01 \\
\hline F4: $8.400 \mathrm{GHz}$ & 22.43 & 22.48 \\
\hline F5: $8.425 \mathrm{GHz}$ & 22.50 & 22.49 \\
\hline F6: $8.450 \mathrm{GHz}$ & 22.58 & 22.49 \\
\hline
\end{tabular}

The results show good correlation between values obtained at extrapolated via analysis from Compact Range of RYMSA and the values obtained with the RF oven test set-up. Standard deviation is around $0.15 \mathrm{~dB}$, which is approximately the uncertainty associated to the planar system. Therefore, no critical degradations are expected neither in the maximum gain figure or radiation pattern within the specified thermal range.

\section{CONCLUSIONS}

MGA-RFA EQM completed successfully the abovedescribed test as part of the qualification campaign. The assumptions for high temperatures that were based on analysis and extrapolations from compact range measurements were validated. Proper performance of the antenna in the full mission environment is ensured thanks to the environment simulation set-up. MGA-RFA PFM has been already manufactured and successfully passed its acceptance campaign, being delivered to SENER for its integration with the rest of the MGAMA PFM.

\section{REFERENCES}

[1] http://sci.esa.int/bepicolombo/

[2] http://www.gr.ssr.upm.es/leha/

[3] IEEE Standards Test Procedures for Antennas. IEEE Std. 149-1979. John Wiley.

[4] G.E. Evans. Antenna Measurement Techniques. Artech House Inc. 1990 